\title{
Determinants of contraceptive method choice in Bangladesh: Male perspectives
}

\author{
Md. Shahidul Islam
}

MSS (thesis) Student, Department of Sociology, Shahjalal University of Science and Technology, Sylhet, Bangladesh.

\begin{abstract}
Family planning programs are considered to be an important way to control the rapid population growth of Bangladesh with the main focus being women. As a male dominant country, the knowledge of, attitude towards, and approval of family planning is largely influenced by the male member of couples in their choice of appropriate contraceptive methods. This paper examined the determinants of current choices in family planning methods in relation to male knowledge, attitude and approval of family planning practices in Bangladesh. A total of 430 married men aged 15-49 years were interviewed in Narsingdi, a district town of Bangladesh. The findings revealed that the men's level of contraceptive knowledge and their attitude to modern contraceptive was high in this area. The findings asserted that the contraceptive prevalence rate among couples was $(62.1 \%)$, with oral pills $(26.51 \%)$ and withdrawals $(6.25 \%)$ being the most preferred modern and traditional methods respectively. The multinomial logistic regression model has been applied to understand the determinants of the choice of contraceptive method. These findings indicated that women in skilled occupation, positive attitude towards modern contraceptives of male, high level of knowledge on contraceptive methods of male, approval of family planning by male, and current living children had significantly more positive effects on using modern contraceptives by the couple. It was also found that couples who desired more children were less likely to use modern contraceptives. Alternatively, the education level of the husband and the desired number of additional children had a negative impact on the use of traditional methods while media exposure, a high level of knowledge on contraceptives, and an approval of family planning had positive impacts on the use of traditional contraceptives over not using any method. The government should increase the funding and availability of family planning programs which promote the use of modern contraceptive methods, especially those that are targeted towards the male population.
\end{abstract}

Keywords: Contraceptive, Family Planning, Male, Knowledge, Attitude, Bangladesh.

\section{Introduction}

In developing countries, the use of contraceptives are increasing and some countries like Bangladesh, Thailand and Indonesia have achieved notable success. ${ }^{1}$ The selection of contraceptive methods depends on psychological, social and cultural factors (i.e. perceptions of contraception and existing social norms about this culture). ${ }^{2}$ Moreover, the use of contraception is largely dependent on the knowledge and attitudes of males towards modern contraception (e.g. contraceptive pills, implants, injectables, intrauterine device, male and female condoms, vasectomy, tubal ligation etc.). ${ }^{3}$ The partner's disapproval of contraceptive use is associated with more use of traditional methods (e.g. coitus interruptus, periodic abstinence etc.). A study in Kenya showed that women whose partners disapproved the use of modern contraception were 1.15 times more likely to use traditional contraceptive methods. ${ }^{4}$ Another study in Bangladesh showed that couples who had more than four children were 2.29 times more likely to use modern contraceptives. ${ }^{5}$ Another study in Bangladesh found that married males, who had positive attitude towards modern contraceptives, were 3.98 times more likely to use any kinds of contraception. ${ }^{3}$

Bangladesh is a small country in Asia with a large population (149 million, 1035 people/sq. $\mathrm{km}$ ) and is ranked the

\section{Practice Points}

- In Bangladesh, the approval of contraceptive methods is largely influenced by the male member of couples.

- Men's contraceptive knowledge and their attitude to modern contraceptive was high in this area.

- The factors which influence the use of modern contraceptive methods include: wife's education and occupation, number of living children in the family, male's higher knowledge and positive attitude towards modern contraceptives, and male's approval of family planning and exposure to media.

- Wife's education, skilled occupation, higher knowledge on contraceptive methods and media exposure were positively associated with use of traditional contraceptive methods.

- The family planning program in Bangladesh should more emphasis to male participation in family planning program and reproductive health.

Correspondence: Md. Shahidul Islam, MSS (thesis) Student, Department of Sociology, Shahjalal University of Science and Technology, Sylhet, Bangladesh. Email: shahidulsoc@gmail.com.

South East Asia Journal of Public Health 2013;3(1):50-56. (C) 2013 Islam, publisher and licensee Public Health Foundation Bangladesh. This is an Open Access article which permits unrestricted non-commercial use, provided the original work is properly cited. 
seventh most populous country in the world. ${ }^{6}$ However, national family planning programs and non-government organizations in Bangladesh are playing an important role in the effort to lower the fertility rate and to increase prevalence of contraceptive use. ${ }^{6}$ Family planning programs in Bangladesh provide their services to households and not only provide information about modern contraceptive methods, but also distributes various forms of contraception in a house to house basis. ${ }^{7}$ The fertility rate in Bangladesh has decreased from 6.3 births per woman in the mid-1970s to 2.7 births in 2007 , and the use of contraception has increased from $7.7 \%$ to $55.8 \%$ during the same period. ${ }^{5}$ The prevalence of contraceptive use reached $61 \%$ in Bangladesh in $2011 .{ }^{8}$ But the contraceptive prevalence rates are not the same in all areas of Bangladesh. The use of general contraceptives are lower (52\%) in rural areas compared to urban $(60 \%)$ areas, as is the use of modern contraceptive methods. ${ }^{9}$ This shows that the social environment around people also influences the choice of contraceptive method. ${ }^{2}$ However, men can play an important role in reproductive decision-making through their sexual, economic and social relationships. ${ }^{10}$

Most of the time, males make the decision of when to have a child, family size, when to stop childbirth, and what types of methods are used for the spacing of births. ${ }^{3}$ The husband's approval of contraceptive use increase the contraceptive prevalence rates while disapproval leads to decrease the rate. ${ }^{10}$ Knowledge of contraception is playing a vital role in the use of different contraceptive methods. ${ }^{11}$ Males prefer to use the methods which they know about well, and those which are easily available to them. ${ }^{12}$ In Bangladesh, oral pills, injections and periodic abstinence are the most preferred methods but the rate of use of male-centered methods (i.e. condoms and male sterilization) is very low. ${ }^{8}$ People generally chose contraceptive methods that are normally used and are socially accepted in their area. ${ }^{13}$ People reject the use of contraceptives which are restricted by social norms ${ }^{13}$ However, the choice of appropriate contraceptives is an important aspect of a couple's reproductive health. ${ }^{12}$ There is great deal of evidence in Bangladesh that males are more likely to use modern methods when they have already reached a desired family size. ${ }^{12}$ Being the primary decision makers during the choice of family planning methods, they are more likely to choose male centered methods and shoulder all the responsibility. ${ }^{12}$ Positive attitudes towards family planning and the approval of the use of modern contraceptive methods are the vital factors that will lead to the reduction of the fertility rate of this country. ${ }^{8}$ As Bangladesh is a male dominated country, the positive attitude of men towards family planning and contraceptive use is more important than women in reproductive decisionmaking.

Many studies ${ }^{3,5,6}$ have been carried out in Bangladesh about the determinants of family planning method choice in relation to various socio-demographic variables of women. But the role of the knowledge, attitude and approval of family planning of men has not been examined. So it is unknown how it affects a couple's choice of contraceptive method. This study examined the factors that influence the choice of contraceptive method choice in relation to male knowledge, attitude and approval of family planning practices in Bangladesh.

\section{Materials and methods}

\section{Participants}

A cross-sectional study was conducted in Narsingdi, Bangladesh using a social survey method to collect data from the respondents. The primary data was collected through face to face interviews from the representative sample of respondents. A two-stage cluster sampling procedure was adopted to select the respondents for the household-based survey. The married men within reproductive age (15-49 years) were selected as the respondents. There are nine wards in Narsingdi town. A ward is a lowest administrative tier of a city or town in Bangladesh that consists of several village or mohallas. ${ }^{14}$ Two wards (Ward 2 and Ward 9) were randomly selected from the nine wards of the town. A list about 1210 of the married men aged 15-49 years were created from these two wards by house to house enumeration. In the second stage, a sample, consisting of 430 married men, was selected by using simple random sampling method.

\section{Data collection}

Closed and open-ended questions were included in the questionnaire, and data was collected through face to face interviews by the researcher himself. The data was collected during May to July in 2012. The questionnaire consisted of two sections: the first section focused on the socio-demographics characteristics of the respondents, and second section focused on the contraceptive practices of the respondent, the types of currently used contraceptive methods, the knowledge, attitude and approval of modern contraceptive methods of the respondents. The respondents willingly participated in the study. The confidentiality of the respondents was maintained because the interviews were conveyed either in private rooms or places so that other people could not overhear.

\section{Variables}

The independent variables of this study were the husband's education, husband's occupation, women's education, women's occupation, currently living children, the desired number of additional children, the knowledge of the males about contraceptives, the attitude of men towards modern contraceptive methods, men's approval of family planning and media exposure. The dependent variable was the family planning method choice. The family planning method choice of this research was measuring currently using of contraceptive method. The dependent variable of contraceptive method choice is classified into three categories: (i) currently using any modern method, (ii) currently using any traditional method, and (iii) currently not using any method. 


\section{Statistical analysis}

The SPSS (Statistical Package for Social Science) 17 program was used to analyze the data. Univariate analysis as frequency distribution was used to analyze and present the background profile of the respondents. Next, in multivariate analysis, the multinomial logistic regression was applied while measuring the effects of the independent variables when choosing modern or traditional methods over not using any method.

Husband approval of family planning was measured by using with a series of six questions assessing approval (yes $=1, n o=0$ ) related to reproductive health issues. Cronbach's alpha for approval of family planning index questionnaires was 0.91 which is standard.

Attitude on modern contraceptive was measure by using six items focused on respondents' attitude on modern contraceptive and these items were rated a 5 point Likert scale. Next, the score summed and ranged from 6 -30. Attitude level was than categorized as negative, medium and positive attitude on contraceptives using the score 6-14, 15-23 and 24-30. Cronbach's alpha found for attitude index questionnaires was 0.87 which is good

Knowledge on contraceptive was measured by asking whether they well known the 10 types of modern contraceptive methods. Next, the variable was categorized into two groups. The score ranges from 0-4 were summed the low knowledge on contraceptives and range from 5-10 were summed the high knowledge on contraception. Cronbach's alpha found for knowledge index questionnaires was 0.75 which was acceptable. So, internal consistency of the reliability of these three indices questionnaires was good.

\section{Results}

Table 1 shows that $80 \%$ of the respondents had formal education. More than $21.4 \%$ passed higher secondary education and $18.4 \%$ took part in secondary educations, but $20 \%$ of the respondents were totally illiterate. Regarding the occupational status of the husband, $52.3 \%$ were unskilled and $36.35 \%$ were skilled, and among them only $11.4 \%$ had professional jobs. A majority of the wives had passed primary examinations (34.2\%) and $69.1 \%$ of wives were not involved in any earning activity. Only $15.4 \%$ of the respondents had no children, while $17.4 \%$ had one child, $26.7 \%$ had two children, $23.7 \%$ had three children, and $16.5 \%$ had four or more children. So the majority of the respondents had intention to keep the family size in two or three children. Most of the respondents (45.1\%) did not want additional children and were more likely to use modern contraceptive methods. A majority of the husbands had high knowledge on $(64.2 \%)$, and a positive attitude towards $(44.2 \%)$ modern contraception, and approved $(73.3 \%)$ such family planning methods. Approximately onequarter of the couples chose pills as their preferred method of family planning. In terms of traditional methods, $3 \%$ used the safe period and $6.3 \%$ used the withdrawal method.
Table 1: Socio-economic characteristics of the respondents

\begin{tabular}{|c|c|}
\hline $\begin{array}{l}\text { Socio-economic } \\
\text { characteristics }\end{array}$ & Respondents (\%) \\
\hline \multicolumn{2}{|l|}{ Male's Education } \\
\hline No education & $86(20 \%)$ \\
\hline Primary & $78(18.1 \%)$ \\
\hline Secondary & $79(18.4 \%)$ \\
\hline Higher secondary & $92(21.4 \%)$ \\
\hline Bachelor & $62(14.4 \%)$ \\
\hline Masters and above & $33(7.7 \%)$ \\
\hline \multicolumn{2}{|l|}{ Male's occupation } \\
\hline Unskilled & $225(52.3 \%)$ \\
\hline Skilled & $156(36.3 \%)$ \\
\hline Professional & $49(11.4 \%)$ \\
\hline \multicolumn{2}{|l|}{ Education of wives } \\
\hline No & $114(26.5 \%)$ \\
\hline Primary & $147(34.2 \%)$ \\
\hline Secondary & $98(22.8 \%)$ \\
\hline Tertiary & $71(16.5 \%)$ \\
\hline \multicolumn{2}{|l|}{ Occupation of wives } \\
\hline Housewife & $297(69.1 \%)$ \\
\hline Unskilled & $68(15.8)$ \\
\hline Skilled & $65(15.1 \%)$ \\
\hline \multicolumn{2}{|l|}{ Current children } \\
\hline 0 & $67(15.6 \%)$ \\
\hline 1 & $75(17.4 \%)$ \\
\hline 2 & $115(26.7 \%)$ \\
\hline 3 & $102(23.7 \%)$ \\
\hline $4+$ & $71(16.5 \%)$ \\
\hline \multicolumn{2}{|c|}{ Desired additional children } \\
\hline 0 & $194(45.1 \%)$ \\
\hline 1 & $107(24.9 \%)$ \\
\hline 2 & $99(23 \%)$ \\
\hline $3+$ & $30(7 \%)$ \\
\hline \multicolumn{2}{|c|}{ Attitude on modern contraceptive } \\
\hline Negative & $78(18.1 \%)$ \\
\hline Medium & $162(37.7 \%)$ \\
\hline Positive & $190(44.2 \%)$ \\
\hline \multicolumn{2}{|c|}{ Knowledge on modern contraceptive } \\
\hline Low knowledge & $154(35.8 \%)$ \\
\hline High knowledge & $276(64.2 \%)$ \\
\hline \multicolumn{2}{|c|}{ Approval of family planning } \\
\hline Approved & $115(26.7 \%)$ \\
\hline Disapproved & $315(73.3 \%)$ \\
\hline \multicolumn{2}{|l|}{ Media exposure } \\
\hline No & $109(25.3 \%)$ \\
\hline Yes & $321(74.7 \%)$ \\
\hline
\end{tabular}

Of the total couples, $50.9 \%$ were using a modern contraceptive method, $10.2 \%$ were using a traditional method, and $38.8 \%$ were not using any method (Table 2 ). In this study, the most commonly used modern contraceptive method was the pill $(28.6 \%)$; other methods included: condoms $(7.2 \%)$ and female sterilization $(6.04 \%)$.

Table 3 shows the multivariate analysis of the effects of male knowledge on contraceptive, approval of family planning, attitude towards modern contraceptive and other socio-economic factors on contraceptive choice. 
Table 2: Types of contraceptive method choice by the couples

\begin{tabular}{|c|c|}
\hline $\begin{array}{c}\text { Contraceptive } \\
\text { methods }\end{array}$ & $\begin{array}{c}\text { Respondents } \\
(\mathbf{\%})\end{array}$ \\
\hline Modern methods & $219(50.9 \%)$ \\
\hline Pill & $114(26.51 \%)$ \\
\hline IUD & $15(3.48 \%)$ \\
\hline Diaphragm/jelly/foam & $3(0.69 \%)$ \\
\hline Condom & $31(7.2 \%)$ \\
\hline Norplant/implant & $2(0.46 \%)$ \\
\hline Female sterilization & $26(6.04 \%)$ \\
\hline Male sterilization & $4(0.93 \%)$ \\
\hline Injection & $24(5.58 \%)$ \\
\hline Traditional methods & $44(10.2 \%)$ \\
\hline Safe period/periodic abstinence & $13(3.02 \%)$ \\
\hline Withdrawal & $27(6.27 \%)$ \\
\hline Postpartum abstinence & $4(0.93 \%)$ \\
\hline Not using any method & $167(38.8 \%)$ \\
\hline
\end{tabular}

In this model, the effects of these factors on the choice of modern methods and traditional methods were compared to not using any method. Men who had positive attitude towards modern contraceptive were 2.73 times more likely to use modern contraceptives than not using any method. It is clear that men who have a positive attitude towards modern contraceptives use more of those than traditional methods.

The approval of family planning by male partner was also associated with using contraceptive method. It was found that men who had approved family planning methods were 2.13 times more likely to use modern contraceptive method. Couples were 2.24 times more likely to use modern contraceptive when husband had high knowledge on modern contraceptive.

Similarly men who had higher knowledge on modern contraceptive were 4.48 times more likely to use traditional methods than not using any method. Highly educated women were more likely to use modern and traditional methods. Couples were 6.44, 3.11 and 3.56 times more likely to use modern methods where women had tertiary, secondary and primary education respectively. Similarly, women who had tertiary education were more likely to use modern methods and traditional methods than not using any method. Husband's education was negatively associated with traditional methods.

Women's working status was a vital factor to use modern contraceptive methods. Skilled women were 2.61 times more likely to use modern contraceptive but unskilled worker women were 0.43 times less likely to use modern contraceptives. It was also demonstrated the men who had media exposure were 1.76 times more likely to use modern methods and 4.94 times more likely to use traditional methods. It was also found that couples who had four or more children were 9.22 times more likely to use modern methods. Most of them were using permanent methods.

\section{Discussion}

The study examined how socio-economic factors affect the use and approval of different contraceptive methods among married men. It was found that the following factors influence the use of modern contraceptive method of couples: wife's education, wife's involvement in economic activities, number of living children in the family, positive attitude towards modern contraceptives, higher level of knowledge on contraceptives, male's approval of family planning, and male's exposure to media. It was also demonstrated that wife's education, skilled occupation, high level of knowledge on contraceptive methods and media exposure were positively associated with use of traditional contraceptive methods.

In family planning, the programs usually aim women as the target group and ignore the role of men. ${ }^{15}$ In traditional society where decisions are largely taken by men, they should be actively involved in reproductive decision of the couple. ${ }^{15,16}$ This study revealed that husband approval of family planning is associated with any kinds of contraceptives. Husband approval of family planning is also important to those women who intend to use modern contraceptives in future. ${ }^{17} \mathrm{We}$ found that attitude of modern contraceptive is also associated with modern contraceptive use but it has no significant effects in choosing traditional methods. Evidence show that positive attitude of couples towards modern contraceptive increases the likelihood of using modern contraceptive. $^{3,16}$

This study also found that women's education and participation in skilled labor play an important role to use modern contraceptives and encourages having small family size. In Bangladesh, the government has set targets to educate women and increase their participation in the labor market. It was also found that that media exposure is positively associated with the use of any kinds of contraceptive. Evidence shows that mass media is an important determinant to change the fertility behavior in Bangladesh. ${ }^{15,18,19}$ Due to their education, occupation and socialization, men are more exposed to radio, TV and newspapers which may have influence on their attitudes to use of family planning methods. ${ }^{15}$ Mass media is the easiest and probably the only way to communicate with the people to inform the consequences of increased population in the country and create awareness of using contraceptive methods. ${ }^{20}$

People avoid the contraceptive methods which need minor surgical intervention. ${ }^{2}$ It has been found that males usually avoid vasectomy as it needs minor surgery. Contraceptive use is mostly dependent on couple's sexual satisfaction; most males are not interested in using condoms because they think that it reduces their sexual pleasure. ${ }^{12}$ Most couples turned to use the pill for spacing birth. ${ }^{21}$ Implant/jelly/foam using rate is so low because it is costly and not available everywhere in Bangladesh. Pills are largely used because of its low cost and availability. Condoms are generally used as family planning method for temporarily spacing birth 
Table 3: Multinomial logistic regression analysis - traditional and modern contraceptive use over not using any method by the respondents

\begin{tabular}{|c|c|c|c|c|}
\hline \multirow[t]{3}{*}{ Variables } & \multicolumn{4}{|c|}{ Methods of contraception used by couples } \\
\hline & \multicolumn{2}{|c|}{ Modern vs. no method } & \multicolumn{2}{|c|}{ Traditional vs. no method } \\
\hline & Odds ratio & $95 \% \mathrm{CI}$ & Odds ratio & $95 \% \mathrm{CI}$ \\
\hline \multicolumn{5}{|l|}{ Male's education } \\
\hline Masters and above & 2.449 & $0.907-4.122$ & $0.003 * * *$ & $0.000-0.198$ \\
\hline Bachelor & 1.566 & $0.661-2.34$ & $0.003 * * *$ & $0.000-0.057$ \\
\hline Higher secondary & 1.122 & $0.886-3.388$ & $0.009 * * *$ & $0.001-0.113$ \\
\hline Secondary & $0.096 * *$ & $0.279-7.498$ & $0.013 * * *$ & $0.001-0.155$ \\
\hline Primary & 0.449 & $0.011-0.835$ & 0.326 & $0.721-1.474$ \\
\hline No education (ref) & 1.000 & & 1.000 & \\
\hline \multicolumn{5}{|l|}{ Male's occupation } \\
\hline Professional & 0.436 & $0.766-0.2 .487$ & 1.034 & $0.07-14.28$ \\
\hline Skilled & 1.051 & $0.319-3.461$ & 1.899 & $0.306-11.78$ \\
\hline Unskilled (ref) & 1.000 & & 1.000 & \\
\hline \multicolumn{5}{|l|}{ Education of wife } \\
\hline Tertiary & $6.436 * *$ & $1.294-19.923$ & $3.003 * *$ & $1.599-9.877$ \\
\hline Secondary & $3.113 * *$ & $1.017-9.533$ & $2.487 * * *$ & $1.055-6.315$ \\
\hline Primary & $3.562 * *$ & $1.361-9.312$ & $4.783 * *$ & $1.242-18.413$ \\
\hline No education (ref) & 1.000 & & 1.000 & \\
\hline \multicolumn{5}{|l|}{ Wife's occupation } \\
\hline Skilled & $2.610^{*}$ & $0.945-7.211$ & $3.691 *$ & $0.845-16.120$ \\
\hline Unskilled & $0.433^{*}$ & $0.180-1.004$ & 0.334 & $0.090-1.236$ \\
\hline Housewife (ref) & 1.000 & & 1.000 & \\
\hline \multicolumn{5}{|l|}{ Current children } \\
\hline $4+$ & $9.216 * *$ & $1.51-20.13$ & 6.205 & $0.427-15.234$ \\
\hline 3 & $3.067 *$ & $0.64-14.67$ & 2.603 & $0.231-14.362$ \\
\hline 2 & $5.304 * *$ & $1.28-16.12$ & 3.117 & $0.304-15.912$ \\
\hline 1 & $3.747 * *$ & $1.04-13.49$ & 2.687 & $0.294-12.247$ \\
\hline 0 (ref) & 1.000 & & 1.000 & \\
\hline \multicolumn{5}{|c|}{ Desired number of additional children } \\
\hline $3+$ & $0.011 * * *$ & $0.001-0.124$ & 0.231 & $0.021-2.500$ \\
\hline 2 & $0.012 * * *$ & $0.003-.046$ & $0.015 * * *$ & $0.002-0.120$ \\
\hline 1 & $0.065 * * *$ & $0.021-0.171$ & $0.064 * * *$ & $0.01-0.231$ \\
\hline 0 (ref) & 1.000 & & 1.000 & \\
\hline \multicolumn{5}{|c|}{ Attitude on modern contraception } \\
\hline Positive & $2.731 *$ & $0.921-8.099$ & 2.937 & $0.714-12.085$ \\
\hline Medium & 2.198 & $0.848-5.696$ & 1.979 & $0.560-6.991$ \\
\hline Negative (ref) & 1.000 & & 1.000 & \\
\hline \multicolumn{5}{|c|}{ Knowledge on modern contraceptive } \\
\hline High knowledge & $2.354 * *$ & $0.953-5.813$ & $4.477 * *$ & $1.313-15.268$ \\
\hline Low knowledge (ref) & 1.000 & & 1.000 & \\
\hline \multicolumn{5}{|c|}{ Husband approve family planning } \\
\hline Approved & $2.125 * *$ & $0.950-5.813$ & $3.355^{* *}$ & $0.940-11.974$ \\
\hline Disapprove (ref) & 1.000 & & 1.000 & \\
\hline \multicolumn{5}{|l|}{ Media exposure } \\
\hline Yes & $1.762 * *$ & $0.757-4.104$ & $4.938 * *$ & $1.473-16.547$ \\
\hline No (ref) & 1.000 & & 1.000 & \\
\hline
\end{tabular}

ref: reference category; $* * * \mathrm{p}<0.01 ; * * \mathrm{p}<0.05 ; * \mathrm{p}<0.10$

and reducing the risk of sexually transmitted diseases. Injectables are also common because the government and NGOs clinics provide these methods for the couples. $^{21}$
It was also shown that the desired number of additional children have negative impacts on using modern and traditional methods. Couples who have desired one, two and three children are less likely to use modern and 
traditional methods. Generally people who plan to take additional children don't use modern methods. So a male's desired number of additional children was negatively associated with contraceptive use. ${ }^{22}$ Working women are more able to make their own decisions about method choice and their intention to keep family size small, and most of them use suitable contraceptive methods. ${ }^{11}$ But when wives were unskilled workers, they were less likely to use modern contraceptives. The contraceptive use rate is low for the couples whose wives were unskilled workers and less educated. So, higher working status of wives was positively associated with modern contraceptive use and lower working status was negatively associated with modern contraceptive use.

A study conducted in Bangladesh by Shahjahan et $a l .{ }^{15}$ found that men having more education, higher income level, and those who access to media were more likely to be involved in reproductive healthcare services. ${ }^{15}$ Educated men have proper knowledge on modern contraceptive. ${ }^{15,23}$ This study reveals that men have considerable knowledge on modern contraceptives, including female methods. Fear of side-effects is one of the reasons for not using modern contraceptive and thus lead them to use traditional methods. ${ }^{16,24}$ It is important to increase the provision of appropriate contraceptive knowledge to enhance male participation in reproductive health. Appropriate policies should be developed by considering the related factors to increase males' contraceptive knowledge and hence increase male participation in family planning and reproductive health in Bangladesh. ${ }^{15,18,23}$

There were some limitations of the study. The researcher compiled the complete list of the all married men age (15-49 years) from those selected wards. Only 1210 married men were successfully enlisted. Some of the men may be missing within the age group from this total list. Besides, the sample size was also small, only (430) married men were included in the study. As a cross-sectional study, causality cannot be established in this study. Longitudinal studies with large sample size should be conducted to examine the determinants of male knowledge, attitude and approval of family planning practices.

\section{Conclusion}

The study revealed that socio-economic factors, male knowledge, attitude and approval of family planning are important for contraceptive method choice. Among the socio-economic factors, males' education, wives' education, wives' occupation, number of living children, desired number of additional children, mass media exposure were associated with contraceptive method choice. But the rate of use of male contraceptive methods is low. So there arises a great inequality to method choice. Family planning programs in Bangladesh should give more emphasis on male participation in family planning program and reproductive health. Radio, television newspaper and other mass media can provide information to encourage male participation and responsibility in contraceptive method choice.

\section{References}

1. Greenspan A. Adding choice to the contraceptive Mix: Lessons from Indonesia. Asia-Pacific Population Policy. No 19. Honolulu: Population Institute of East-West Centre, 1991.

2. Olaitan OL. Factors influencing the choice of family planning among couples in Southwest Nigeria. Int J Med Med Sci 2011;3(7):227-32.

3. Kamal MM, Islam MS, Jalil M. Determinants of current contraceptive use among married couples in Bangladesh: Views of males. Asian Profile 2013;42(2),161-72.

4. Magadi AM, Curtis LS. Trends and determinants of contraceptive method choice in Kenya. Stud Fam Plann 2003; 34(3):149-59.

5. Kamal SMM. Contraceptive use and method choice in urban slum of Bangladesh. Conference paper. International Conference on Family Planning: Research and Best Practices, Kampala, 1518 Nov, 2009.

6. Rahman MD, Mondal MNI, Ali MK. A study on the factors affecting the use of contraception in Bangladesh. Int Res $J$ Biochem Bioinformatics 2011;7:178-83.

7. Randall E. Family Planning Programmes Review. London: Population Matters, 2012.

8. The World Bank. Contraceptive Prevalence (\% of women ages 15-49). http:// data.worldbank.org/indicator/SP.DYN.CONU. $\underline{\text { ZS }}$ (accessed June 2013)

9. Rahman MM, Islam AZ, Islam MR. Rural-urban differentials of knowledge and practice of contraception in Bangladesh. J Popul Soc Stud 2010;18:87-110.

10. Abraham W, Adamu A, Deresse D. The Involvement of Men in Family Planning: An Application of Transtheoretical Model in Wolaita Soddo Town South Ethiopia. Asian J Med Sci 2010;2:44-50.

11. Parveen SS. Factors Affecting contraceptive use among on married female adolescents in Bangladesh. [thesis]. Bangkok: Mahidol University, 2000 .

12. Donahoe D. Men and Family Planning in Bangladesh: A review of the literature. Final report. Asia and Near East Operation Research and Technical Assistance Project, The Population Council, Bangladesh, 1996.

13. Gayen K, Raeside R. Social networks and contraception practice of women in rural Bangladesh. Soc Sci Med 2010; 71(9):1584-92. 
14. Wikipedia, the free encyclopedia, Wards of Bangladesh. http://en.wikipedia.org/wiki/ Wards_of_Bangladesh (accessed June 2013)

15. Shahjahan M, Mumu SJ, Afroz A, Chowdhury HA, Kabir R, Ahmed K. Determinants of male participation in reproductive healthcare services: a cross-sectional study. Reprod Health 2013;10(1):27.

16. Ankomah A, Anyanti J, Adebayo S, Giwa A. Barriers to Contraceptive Use among Married Young Adults in Nigeria: A Qualitative Study. Int J Trop Disease Health 2013;3(3): 267-82.

17. Kamal N. The influence of husbands on contraceptive use by Bangladeshi women. Health Policy Plan 2000.15(1):43-51.

18. Islam MA, Padmada SS, Smith PWF. Degree and Determinants of Men's Contraceptive Knowledge in Bangladesh. Conference Paper. British Society for Population Studies Annual Conference, 2004.

19. Kabir MM, Islam A. The impact of mass media family planning programmes in current use of contraception in urban Bangladesh. J Biosoc Sci 2000; 32:411-9.

20. Rabbi AMF. Mass Media Exposure and its Impact on Fertility: Current Scenario of Bangladesh. J Sci Res 2012; 4(2):383-95.

21. Routh S, Thwin AA, Kane TT, Baqui HA. Userfees for Family-planning Methods: An Analysis of payment behaviour among urban contraceptors in Bangladesh. J Health Popul Nutr 2000;18 (2):69-78.

22. Hossain MB, Phillips JF, Mozumder AB. The effect of husband's fertility preferences on couple's reproductive behavior in rural Bangladesh. $J$ Biosoc Sci 2009;39: 745-57.

23. Kamal MM, Islam MS, Alam MS, Hassan, ABME. Determinants of male involvement in family planning and reproductive health in Bangladesh. Am J Hum Ecol 2013;2(2):83-93.

24. Fakeye O, Babaniyi O. Reasons for non-use of family planning methods at Ilorin, Nigeria; male opposition and fear of methods. Trop Doct 1989;1:114-7. 